Short Review

\title{
Neck Endocrine Surgery in the Era of COVID-19 Surgical Decision Making and Operative Execution
}

\author{
Phillip K Pellitteri, DO, MHA, FACS*, Nicholas Purdy, DO, FACS, Timothy Lindemann, MD, FACS, FAAOA, \\ Thorsen Haugen, MD and Thomas L Kennedy, MD, FACS
}

Department of Otolaryngology/Head \& Neck Surgery, Geisinger Health System, Danville, PA, USA

\begin{abstract}
Objective: To describe recommendations for the decision making and management of patients with surgical neck endocrine disease during the COVID-19 pandemic.

Data sources: Review of relevant literature based on standards of surgical practice, together with surgical trends at major institutions with high volume neck endocrine surgery. These elements are contrasted with the current pandemic environment and its effect on accepted surgical practice.

Review methods: Patients with surgical endocrine disorders related to the thyroid and parathyroid glands, will require stratification for treatment as determined by severity of disease and recalcitrance to medical therapy in an environment which conserves healthcare related resources and possesses limited viral testing capability.

Conclusion: Stratification of treatment decision making and execution for patients with surgical neck endocrine disorders will involve both patient related elements and health care environmental conditions related to the pandemic involving: Disease entity, health care resources, facility capability and availability of viral testing.

Implications for practice: Patients with surgical neck endocrine disorders may be managed according to risk, based on both patient and pandemic related health environment issues, in determining the need and timing for surgical intervention. A decision algorithm is offered as a platform for discussion and consideration.
\end{abstract}

Keywords

COVID, Thyroid, Parathyroid, Pandemic, Thyroidectomy

\section{Introduction}

The onset and rapid progression of the novel coronavirus, SARS-CoV-2, pandemic has created a widely variable viewpoint in decision making and execution in a variety of surgical disorders. These views range from elimination of all elective procedures while providing urgent surgical services to following full surgical schedules without interruption in service [1]. Influences on these different policies are multifactorial, and include: Testing capability, infection rates, available PPE, organizational resources, and the need to balance economic forces with surgical care delivery in high risk populations. Otolaryngologists/head and neck surgeons are at the highest risk of exposure and potential for infection, owing to the fact that the greatest viral density is found in the nasal cavity/nasopharynx, and manipulation of these anatomic areas thru diagnostic and therapeutic procedures carries with it possible aerosolization of viral particles within droplets during upper aerodigestive tract (UADT) procedures/surgery [2]. Apart from procedures involving the UADT, other neck procedures which do not directly involve high risk anatomic areas, may involve, directly or indirectly, portions of the aerodigestive system, and thus raise the risk of exposure. We offer in this presentation, recommendations for decision making and surgical management of neck endocrine disorders during the viral pandemic, relative to: Specific disorders and the urgency for surgery, alternative non-surgical management, risk assessment with respect to viral exposure, and technical surgical nuances.

\section{Thyroid Disease}

\section{Benign disorders}

Nodular thyroid disease, including multinodular thyroid glands, dominant nodules which are cytologically benign, thyromegaly and diffuse thyroid enlargement should be evalu-

*Corresponding author: Phillip K Pellitteri, DO, MHA, FACS, Department of Otolaryngology/Head \& Neck Surgery, Geisinger Health System, Danville, PA, USA

Received: May 07, 2020

Accepted: June 18, 2020

Published online: June 20, 2020

Citation: Pellitteri PK, Purdy N, Lindemann T, et al. (2020) Neck Endocrine Surgery in the Era of COVID-19 Surgical Decision Making and Operative Execution. J Surgical Endocrinol 2(1):2125 
ated with imaging to assess baseline dimensions and characteristics. Ultrasound should be employed as the mainstay for assessment, reserving cross sectional imaging (CT) for cervico-mediastinal thyroid glands. Situations involving large cervico-mediastinal glands should be assessed for aerodigestive compression and signs of malignancy including, lymphadenopathy or visceral invasion. Unless frank signs of malignancy are present, or there is risk of impending airway compromise, surgery should be delayed for these entities until such time as resources and the widespread availability of viral testing allows.

Benign functional disorders resulting in hyperthyroidism (Grave's disease, toxic adenoma) should be evaluated and risk assessed on a patient by patient basis, recognizing the need to limit non-urgent outpatient visits. If possible, radio iodine therapy should be employed if treatment demands are needed immediately. Patients with disease refractory to

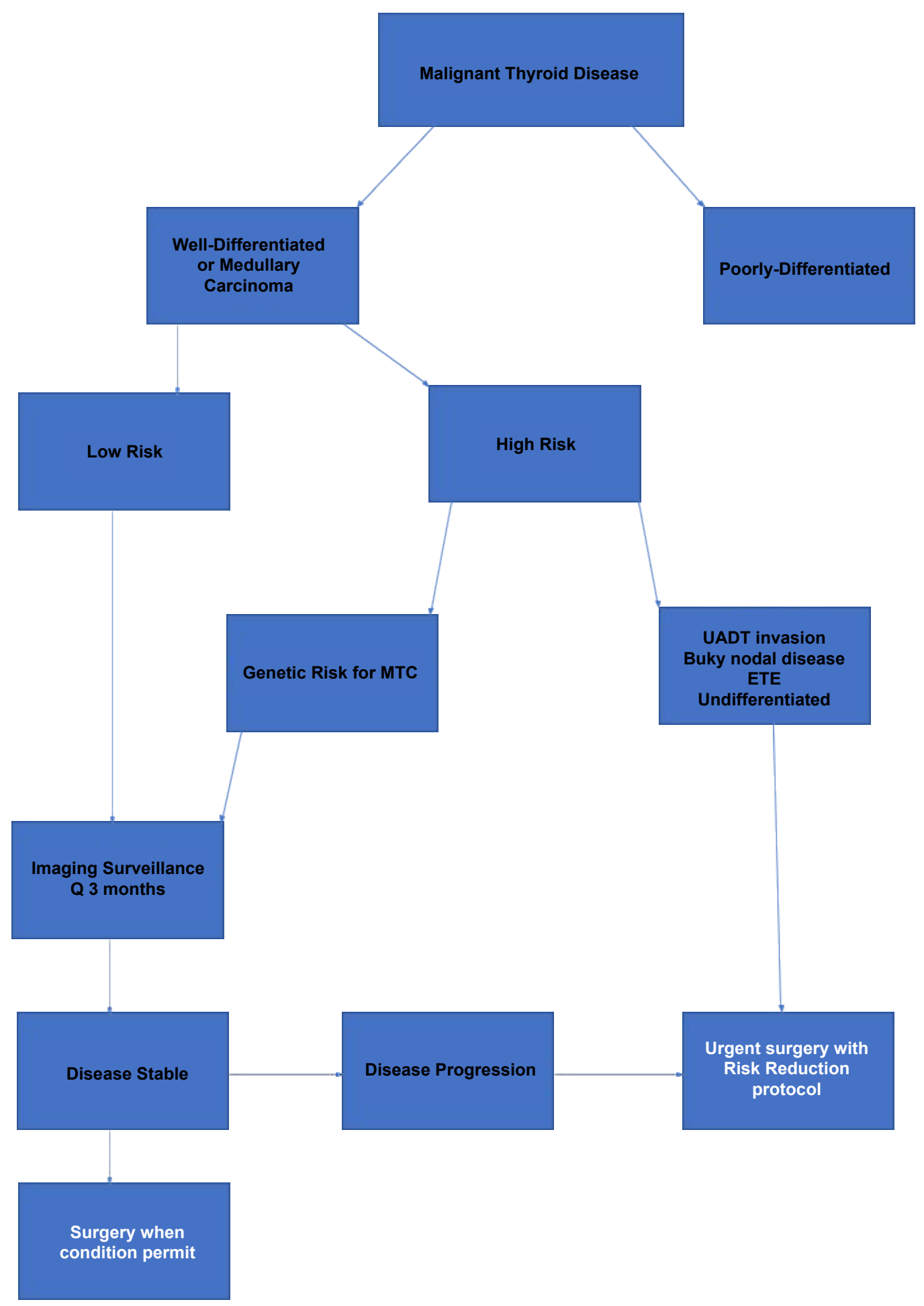

Figure 1: Decision algorithm for benign thyroid disorders. 
medical therapy, or those with ophthalmopathy not amenable to radioiodine, should be managed with antithyroid medication until such time as surgery becomes feasible within the setting of the outbreak. It should be recognized that a small percentage of patients may exhibit agranulocytosis as a result of methimazole use, and that this may mimic symptoms of COVID-19 infection. Patients with medically uncontrolled hyperthyroidism, at risk for thyroid storm, will need to be managed surgically with preoperative viral testing if possible. In the event testing is not available or approved, the highest level of PPE (N95 mask, face shield, powered air purifying respirators, etc.) available should be utilized and the procedure carried out utilizing universal precautions philosophy. All patients ultimately selected for elective surgery should undergo preoperative viral risk assessment, including: Exposure screening, chest radiograph, and PCR swab testing for COVID-19 [3] Figure 1.

\section{Malignant disorders}

Proven malignancy may be separated into high and low risk patients, based on the cytologic findings, stage of disease, and viral risk of exposure for health care workers relative to tumor characteristics. Low risk patients include those in whom cytology yields differentiated thyroid cancer, absence of extrathyroidal extension, lymphadenopathy is not present, and the patient judged to be compliant with imaging surveillance. It would appear prudent to delay surgery in these scenarios until viral testing and resources permit surgery to be performed. Imaging surveillance in these circumstances is important, and we recommend 3-month ultrasound evaluations, or more urgent evaluations, for any change which may suggest advancing tumor. We would consider those patients awaiting completion surgery after partial thyroidectomy for patients in whom cancer was present, and in whom radio iodine is recommended, to be included in this low risk cohort of patients. Active surveillance of patients with biopsy proven differentiated thyroid cancer, with low risk characteristics, is an increasingly acceptable option for those patients with this level of disease $[4,5]$. Active surveillance requires the need for regular surveillance modalities, including imaging, and our protocol calls for interval assessments at 3 months by ultrasound imaging. Surveillance results demonstrating change in primary tumor or the development of metastatic disease call for more urgent evaluation and consideration of surgery.

High risk patients include those with the following clinical elements: Differentiated thyroid cancer with image and/or biopsy evidence of lymph node metastasis or extrathyroidal extension of tumor, thyroid cancer with imaging or clinically proven aerodigestive tract invasion, cytology proven undifferentiated thyroid carcinoma, and medullary thyroid carcinoma with lateral neck metastatic disease. Patients in this stratification will benefit from planned surgical intervention including resection of the primary with compartmentally directed (selective vs. modified radical) lymph node dissection based upon anatomic levels involved. Direct evidence of aerodigestive tract invasion would qualify this scenario as requiring urgent surgery, whereas the remainder would warrant surgical procedures which should not be unduly delayed. We do not believe that patients with hereditary risk of medullary carcinoma based on genetic testing or presence of MEN syndrome, absent proven lymph node metastasis, are candidates for urgent surgical treatment Figure 2.

\section{Parathyroid Disease}

Hyperparathyroidism, in general, is an indolent disease process that does not demand immediate surgical treatment. Clinical scenarios which represent consideration for more urgent surgery include: Hypercalcemia unresponsive to medical therapy with physiologic decompensation, systemic calciphylaxis related to renal induced hyperparathyroidism, and evidence suggesting parathyroid carcinoma. These are several examples of physiologic and oncologic conditions that, in select circumstances, represent indications warranting surgical intervention.

\section{Technical Points}

Recognizing the need for the implementation of surgical techniques needed to minimize potential exposure in the operating room for viral status unknown patients, the following are offered for consideration: Optimizing PPE for procedures which may potentially involve thyroid malignancy invading the aerodigestive system, and avoiding the use of energy instrumentation in the conduct of tumor dissection and ablation in order to reduce potential exposure to the plume created. Rapid sequence induction, together with the use of a protective curtain or negative pressure hood, is recommended at intubation by anesthesia to reduce exposure to aerosolized secretions harboring viral particles [6]. Further, we postulate that the use of endotracheal tube based neuromonitoring during neck endocrine procedures will potentially increase the risk of environmental viral dissemination as a result of the need to properly position the endotracheal tube within the larynx. This is primarily due to increased exposure to the concentrated viral environment within the pharynx. Positioning of the endotracheal tube often requires the need to deflate and then re-inflate the tube cuff, thus creating the potential for further aerosolization of viral particles in the pharynx due to the airflow created after cuff deflation [7]. We recommend monitoring only be implemented in such situations where the need is great due to overall tumor burden, primary location in the central neck, and presence of central compartment lymphadenopathy warranting central compartment neck dissection. The use of a hand-held stimulator for recurrent nerve stimulation with palpation of the posterior cricoarytenoid muscle, represents a potential alternative in selected situations [8]. If parathyroid surgery must be performed for physiologic reasons, we recommend that monitoring not be utilized.

\section{Patient Education}

Many patients, especially those with a cancer diagnosis, have anxiety involving their diagnosis. It is important to discuss the increased potential of COVID-19 exposure to the patient in a hospital environment. Educating them on the low risk of active surveillance of thyroid malignancy in appropriately selected instances, and how this is balanced against the risk of viral exposure, is important in helping to allay their 


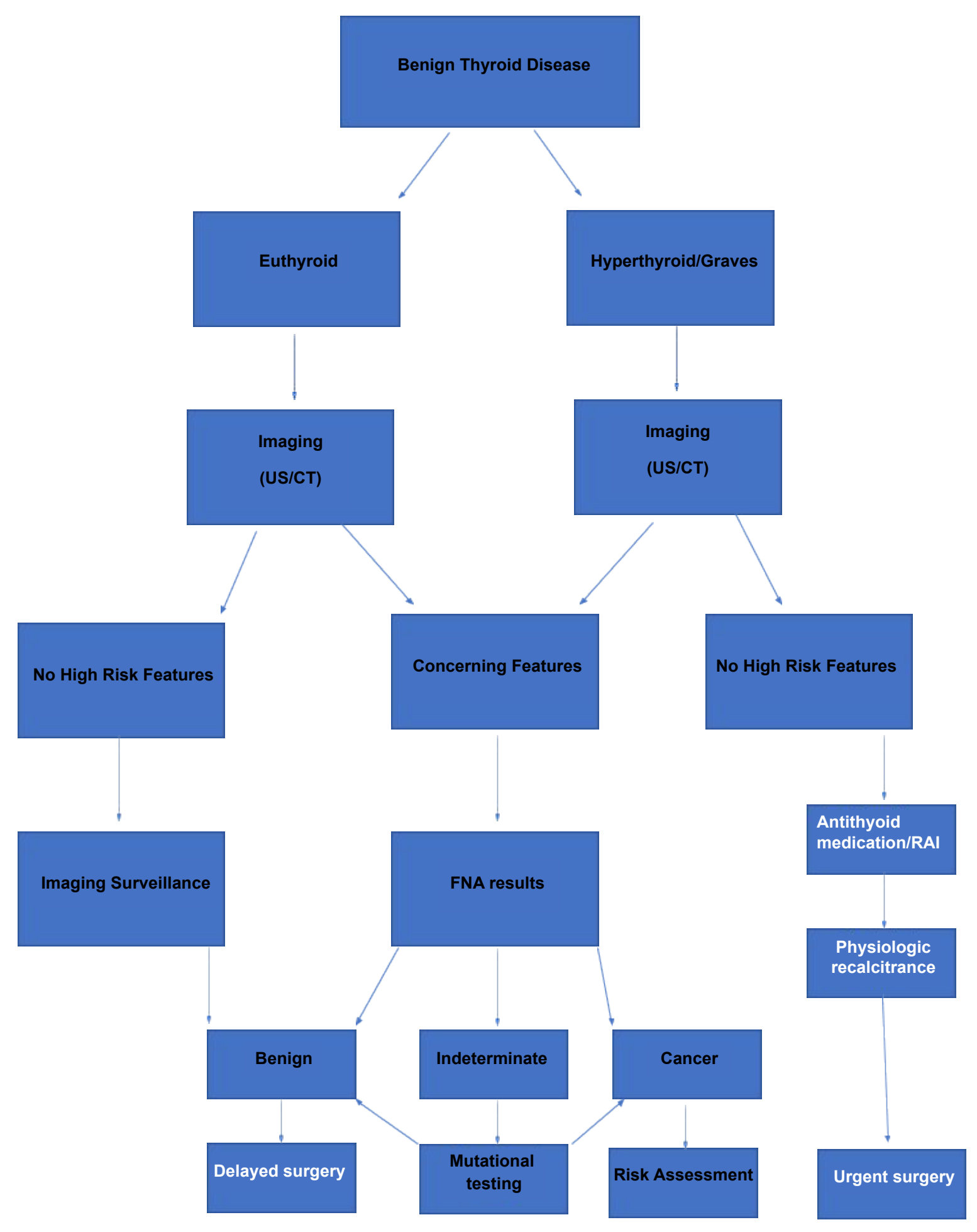

Figure 2: Decision algorithm for malignant thyroid disorders.

anxiety. The importance of mitigation strategies and the need for expanded viral testing protocols should be emphasized in how future considerations regarding surgical urgency are determined.

\section{Surgical Risk Reduction Protocol}

When surgery is required, we implement the following to reduce risk of exposure:
1) Two-week pre-operative home quarantine

a) Daily symptom checks

b) Daily temperature checks

2) Pre-operative chest $X$-ray

3) Proper PPE use (N95 respirator/face shield)

4) Oro/nasopharyngeal decolonization with Chlorhexidine 


\section{Summary}

In the current environment related to the novel SARSCoV-2 pandemic, the operative treatment for patients with potential surgical disease, in all disciplines, has been a source of debate and concern. This results from consideration of risk of exposure to the virus, resource utilization, availability of viral testing, and the overall organization policy related to patient care delivery and the fiscal environment relative to health resources, financial stability, and manpower, during the crisis. With an increased risk of viral exposure for head and neck surgeons, we feel these changes are also relevant to neck endocrine surgery. In this discussion, we offer recommendations on how patients who are candidates for thyroidectomy/parathyroidectomy should be prioritized for surgical treatment based on a patient related risk assessment. We recognize that these recommendations represent, to a great extent, a moving target, as the current environment is quite fluid and continually evolving relative to availability of viral testing and expenditure of resources. Hopefully, the guidance offered will serve as a platform for further inquiry, providing a nidus for additional investigation and recommendations.

\section{References}

1. Multi institutional personal communication.
2. Workman AD, CarterBS, Holbrook EH, et al. (2020) Endonasal instrumentation and aerosolization risk in the era of COVID-19: Simulation, literature review, and proposed mitigation strategies. Int Forum Allergy Rhinol.

3. Coccolini F, Perrone GG, Chiarugi M, et al. (2020) Surgery in COVID-19 patients: Operational directives. World J Emerg Surg 15: 25.

4. Haugen BR (2017) 2015 American Thyroid Association Management guidelines for adult patients with thyroid nodules and differentiated thyroid cancer: What is new and what has changed? Cancer 123: 372-381.

5. Tuttle RM, Fagin JA, Minkowitz G, et al. (2017) Natural history and tumor volume kinetics of papillary thyroid cancers during active surveillance. JAMA Otolaryngol Head Neck Surg 143: 1015-1020.

6. Tang L, Wang J (2020) Anesthesia and COVID-19: What we should know and what we should do. Semin Cardiothorac Vasc Anesth 24: 127-137.

7. Mick P, Murphy R (2020) Aerosol-generating otolaryngology procedures and the need for enhanced PPE during the COVID-19 pandemic: A literature review. J Otolaryngo Head Neck Surg 49: 29.

8. Malik R, Linos D (2016) Intraoperative neuromonitoring in thyroid surgery: A systematic review. World J Surg 40: 2051-2058. 